\title{
Numerical Simulation of the Field Velocities and Local Disturbances of a Long Gravity Wave Passing above an Immersed Vertical Barrier
}

\author{
Laouar Abdelhamid ${ }^{1}$ and Guerziz Allaoua ${ }^{2}$ \\ ${ }^{1}$ Department of Mathematics, University of Annaba, P.O. Box 12, 23000 Annaba, Algeria \\ ${ }^{2}$ Department of Physics, University of Annaba, P.O. Box 12, 23000 Annaba, Algeria \\ Correspondence should be addressed to Laouar Abdelhamid, laouarhamid@yahoo.com
}

Received 20 October 2007; Revised 21 July 2008; Accepted 26 August 2008

Recommended by Roger Grimshaw

This work is interested in the study of the passage of a long gravity wave above an immersed vertical barrier. The latter is placed at a right angle in the middle of the occupied fluid domain which is limited vertically by both a free surface and an impermeable horizontal bottom. We want to determine the field velocity and the local disturbances in the vicinity of the barrier. For this, we use the generalized theory of shallow water and complex variables method. For illustration, we consider a solitary wave as an emitted long wave.

Copyright (c) 2008 L. Abdelhamid and G. Allaoua. This is an open access article distributed under the Creative Commons Attribution License, which permits unrestricted use, distribution, and reproduction in any medium, provided the original work is properly cited.

\section{Introduction}

The problem ofthe passage of gravity waves above an immersed obstacle was studied by several authors (cf. [1-5]). Thus we mention some results already obtained in this field. Dean's work [2] is classified in the theory of the short waves; it concerns particularly the determination of the coefficients of reflection and transmission of the waves passing a completely immersed obstacle (e.g., a barrier). Gulli [4] has studied a same type of obstacle but in the case of the long waves and hehas concluded, at the first order of approximation, that there is not a reflected wave by the obstacle. The studies of Seabra-Santos et al. [5] concern the deformation and the dephasing of the free surface in shallow water due to the solitary mutual interactions or with an isolated obstacle. The theoretical and experimental contributions of Barthélemy et al. [1] concern the phenomena resulting from the internal long waves. In [6], the study of a passage of a long wave over vertical barrier, Germain's shallow water theory associated with the complex variable method has been used to determine the flow.

Our objective in the present paper is to apply the shallow water theory and complex variable method in order to determine the local disturbances at the vicinity of the obstacle 


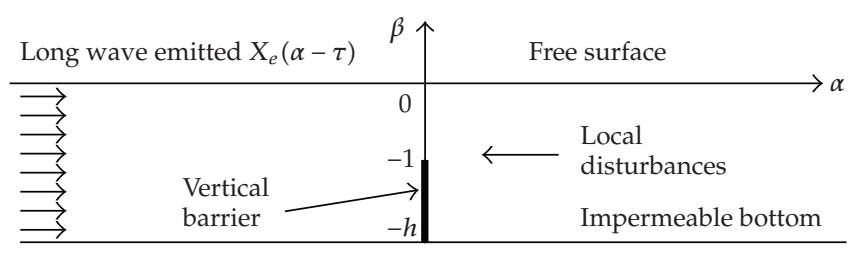

Figure 1: Local disturbances at vicinity of the barrier.

and simulate the velocity field. So, we would like to know particularly with accuracy the kinematic of the flow at the vicinity of the obstacle while the passage of the long gravity wave above the thin immersed vertical barrier. For illustration, we consider a solitary wave generated at the upstream by a piston wave maker.

The plan of this paper is as follows. Section 2 contains two parts: the first part describes the phenomenon, the second part gives general equations and mathematical model. Section 3 gives a determination of the system of equations and the technique of resolution. The last section presents an application and a numerical simulation.

\section{Position of the problem}

\subsection{Description of the phenomenon}

We consider a fixed $O x y$ reference system, where the axis $O y$ is vertically ascendant and the axis $O x$ coincides with the initial free surface. The position of the fluid particle at the moment $t, t>0$, is denoted by $(x, y)$ and their coordinates at the initial position by $(a, b)$, where $a, b$, and $t$ are the variables of Lagrange. Now we introduce new components $X$ and $Y$ as follows:

$$
\begin{aligned}
& X(a, b, t)=x(a, b, t)-a, \\
& Y(a, b, t)=y(a, b, t)-b .
\end{aligned}
$$

The assumption of the shallow water theory (see [3]) introduces distortion space and temporal variables, translating the difference in scale between the horizontal and vertical sizes. This distortion will be characterized by the small parameter which is dependent on the relative long wave amplitude. Thus

$$
\alpha=\varepsilon a, \quad \beta=b, \quad \tau=\varepsilon \sqrt{g h} \cdot t,
$$

where $\sqrt{g h}$ represents the critical celerity ofthe propagated long waves ( $h$ and $g$ are depth of fluid at rest and gravity, resp.).

The description of the phenomenon is as follows: we consider the domain $D$ occupied by fluid of an infinite horizontal band which is limited vertically by a free surface $\beta=0$ and an impermeable horizontal bottom $\beta=-h$. A vertical, thin, and impermeable barrier is placed in the middle of the occupied fluid at right angle to the bottom. The top of the barrier is defined by $(\alpha=0 ; \beta=-l)$. A data long wave $X_{e}(\alpha-\tau)$ emitted upstream passes above the obstacle by creating local disturbances inits vicinity (see Figure 1 ). 


\subsection{General equations and mathematical model}

General equations and mathematical model are listed below:

(i) the kinematic condition expresses the incompressibility of fluid

$$
\frac{\partial Y}{\partial \beta}+\varepsilon\left[\frac{\partial X}{\partial \alpha}+\frac{\partial X}{\partial \alpha} \frac{\partial Y}{\partial \beta}-\frac{\partial X}{\partial \beta} \frac{\partial Y}{\partial \alpha}\right]=0
$$

(ii) the dynamic condition for an irrotational movement

$$
\frac{\partial^{2} X}{\partial \beta \partial \tau}+\varepsilon\left[\frac{\partial X}{\partial \alpha} \frac{\partial^{2} X}{\partial \beta \partial \tau}-\frac{\partial X}{\partial \beta} \frac{\partial^{2} X}{\partial \alpha \partial \tau}+\frac{\partial Y}{\partial \alpha} \frac{\partial^{2} Y}{\partial \beta \partial \tau}-\left(1+\frac{\partial Y}{\partial \beta}\right) \frac{\partial^{2} Y}{\partial \alpha \partial \tau}\right]=0 ;
$$

(iii) the impermeability boundary conditions

$$
\begin{gathered}
\frac{\partial Y}{\partial \alpha}+\varepsilon h \frac{\partial^{2} X}{\partial \tau^{2}}+\varepsilon^{2} h\left[\frac{\partial X}{\partial \alpha} \frac{\partial^{2} X}{\partial \tau^{2}}+\frac{\partial Y}{\partial \alpha} \frac{\partial^{2} Y}{\partial \tau^{2}}\right]=0 \quad \text { at the free surface }(\beta=0), \\
Y(\alpha, \beta, \tau)=0, \quad \text { at the bottom }(\beta=-h), \\
X(\alpha, \beta, \tau)=0, \quad \text { on the obstacle; }
\end{gathered}
$$

(iv) the initial conditions

$$
X(\alpha, \beta,-\infty)=0, \quad Y(\alpha, \beta,-\infty)=0 \quad(\text { at rest })
$$

The resolution of (2.3)-(2.6) requires that the solutions take into account the interaction fluid-obstacle. According to the shallow water theory (see Germain [3]), the solution will be calculated under the entire series in $\varepsilon$ :

$$
\begin{aligned}
& X(\alpha, \beta, \tau)=\sum_{n=1}^{\infty} \varepsilon^{n}\left[X_{n, 0}(\alpha, \beta, \tau)+\sum_{m=1}^{\infty} X_{n, m}(\alpha, \beta, \tau) \exp \left(-\frac{m \lambda \alpha}{\varepsilon}\right)\right], \\
& Y(\alpha, \beta, \tau)=\sum_{n=1}^{\infty} \varepsilon^{n}\left[Y_{n, 0}(\alpha, \beta, \tau)+\sum_{m=1}^{\infty} Y_{n, m}(\alpha, \beta, \tau) \exp \left(-\frac{m \lambda \alpha}{\varepsilon}\right)\right],
\end{aligned}
$$

where $\lambda$ is a determining constant.

The double sum $(m \neq 0)$ in the formula (2.7) characterizes the local disturbances whose amplitude decreases exponentially with the distance. The technique of resolution consists to inject these series in the general equations and to write that they are satisfied with the order $(n, m)$ desired. For example, at the first order of approximation (i.e., $n=1)$, the components $X_{n, m}$ and $Y_{n, m}$ for $m=0$ are given as

$$
\begin{aligned}
& X_{1,0}(\alpha, \beta, \tau)=X_{1,0}(\alpha, \tau), \\
& Y_{1,0}(\alpha, \beta, \tau)=0,
\end{aligned}
$$




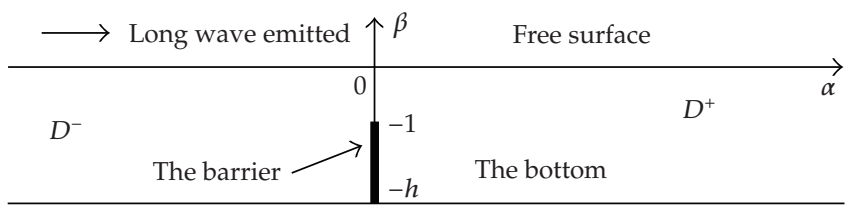

Figure 2: Domains $D^{+}$and $D^{-}$.

and for any $m \neq 0$,

$$
\begin{gathered}
X_{1, m}(\alpha, \beta, \tau)=A_{1, m}(\tau) \cos m \lambda \beta, \\
Y_{1, m}(\alpha, \beta, \tau)=A_{1, m}(\tau) \sin m \lambda \beta .
\end{gathered}
$$

\section{Local disturbances (case the obstacle is a barrier)}

\subsection{System of equations}

At the first order of approximation $(n=1)$, we will calculate the coefficient of the local disturbances. for this, we process as follows. We divide the domain $D$ into two parts $D^{-}=$ $\{-h \leq \beta \leq 0 ; \alpha<0\}$ and $D^{+}=\{-h \leq \beta \leq 0 ; \alpha>0\}$ (see Figure 2). On each domain $D^{-}$or $D^{+}$, according to the point of view of Lagrange, the horizontal and vertical components of the displacement of particle $\left(X_{1}^{-}, Y_{1}^{-}\right)$and $\left(X_{1}^{+}, Y_{1}^{+}\right)$, respectively, can be written as follows:

$$
\begin{aligned}
& D^{-}\left\{\begin{array}{l}
X_{1}^{-}(\alpha, \beta, \tau)=X_{e}(\alpha-\tau)+\sum_{m=1}^{\infty} A_{1, m}^{-}(\tau) \cos m \lambda^{-} \beta \cdot \exp \left(-\frac{m \lambda^{-} \alpha}{\varepsilon}\right), \\
Y_{1}^{-}(\alpha, \beta, \tau)=\sum_{m=1}^{\infty} A_{1, m}^{-}(\tau) \sin m \lambda^{-} \beta \cdot \exp \left(-\frac{m \lambda^{-} \alpha}{\varepsilon}\right)
\end{array}\right. \\
& D^{+}\left\{\begin{array}{l}
X_{1}^{+}(\alpha, \beta, \tau)=X_{e}(\alpha-\tau)+\sum_{m=1}^{\infty} A_{1, m}^{+}(\tau) \cos m \lambda^{+} \beta \cdot \exp \left(-\frac{m \lambda^{+} \alpha}{\varepsilon}\right), \\
Y_{1}^{+}(\alpha, \beta, \tau)=\sum_{m=1}^{\infty} A_{1, m}^{+}(\tau) \sin m \lambda^{+} \beta \cdot \exp \left(-\frac{m \lambda^{+} \alpha}{\varepsilon}\right) .
\end{array}\right.
\end{aligned}
$$

$A_{1, m}^{-}$and $A_{1, m}^{+}$are unknown functions depending on the variable $\tau$, characterizing the amplitude of local disturbances in the domains $D^{-}$and $D^{+}$, respectively, and $\lambda^{-}$and $\lambda^{+}$are given coefficients (we take here $\lambda^{+}=-\lambda^{-}=\lambda=\pi / h$ ). We note that the approximation equations of the phenomenon have been written at the first order of approximation $(1, m)$.

Both the continuity conditions of the flow at the border of the two domains and the impermeability condition of the barrier imply that

$$
\begin{gathered}
X_{1}^{-}(0, \beta, \tau)=X_{1}^{+}(0, \beta, \tau), \quad \alpha=0,-h \leq \beta \leq 0, \\
Y_{1}^{-}(0, \beta, \tau)=Y_{1}^{+}(0, \beta, \tau), \quad \alpha=0,-l \leq \beta \leq 0, \\
X_{1}^{+}(0, \beta, \tau)=0, \quad \alpha=0,-h \leq \beta \leq-l .
\end{gathered}
$$


The condition (3.3a) applied to (3.1) and (3.2) gives the equality

$$
A_{1, m}^{-}(\tau)=A_{1, m}^{+}(\tau)=A_{1, m}(\tau)
$$

The latter expresses the symmetry of the local disturbances at the vicinity of the barrier.

The continuity conditions (3.3a) and (3.3b) of the flow on the segment $[\alpha=0 ;-l \leq$ $\beta \leq 0$ ] give the following equations:

$$
\begin{gathered}
\sum_{m=1}^{\infty} A_{1, m}(\tau) \sin m \frac{\pi \beta}{h}=0, \quad \alpha=0,-l \leq \beta \leq 0, \\
X_{e}(-\tau)+\sum_{m=1}^{\infty} A_{1, m}(\tau) \cos m \frac{\pi \beta}{h}=0, \quad \alpha=0,-l \leq \beta \leq 0 .
\end{gathered}
$$

By considering the linearity of (3.6), if $X_{e}(-\tau) \neq 0$, we have

$$
\begin{array}{r}
1+\sum_{m=1}^{\infty} a_{1, m} \cos m \frac{\pi \beta}{h}=0, \\
\text { where } a_{1, m}=\frac{A_{1, m}(\tau)}{X e(-\tau)} \text { is a constant. }
\end{array}
$$

The problem leads us to solve the following system:

$$
\begin{gathered}
1+\sum_{m=1}^{\infty} a_{1, m} \cos m \frac{\pi \beta}{h}=0, \quad \alpha=0,-h \leq \beta \leq-l, \\
\sum_{m=1}^{\infty} a_{1, m} \sin m \frac{\pi \beta}{h}=0, \quad \alpha=0,-l \leq \beta \leq 0 .
\end{gathered}
$$

Thus the coefficients $a_{1, m}$ are obtained starting from these two time-independent equations.

\subsection{Technique of resolution}

In the domain $D^{+}$, we consider two relations (3.2) of the components $X_{1}^{+}$and $Y_{1}^{+}$and (3.8). We can then construct an analytic complex variable function $z=(\alpha / \varepsilon-i \beta)$ such that

$$
f^{+}\left(\frac{\alpha}{\varepsilon}-i \beta\right)=1+\sum_{m=1}^{\infty} a_{1, m} \exp \left(-m \frac{\pi}{h}\left(\frac{\alpha}{\varepsilon}-i \beta\right)\right) .
$$

This function bounded in the domain $D^{+}$except at the singular points satisfies the impermeability condition at the free surface $\beta=0$ and the bottom $\beta=-h$. Therefore,

$$
Y^{+}(\alpha, \beta)=\operatorname{Im} f^{+}\left(\frac{\alpha}{\varepsilon}-i \beta\right)=0, \quad \text { on the boundaries } \beta=0, \beta=-h,
$$




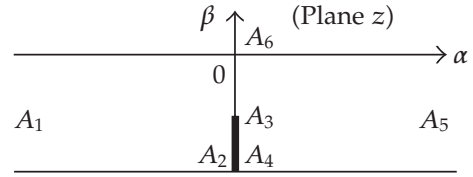

(a)

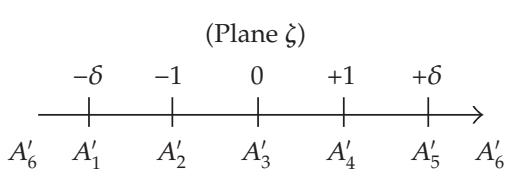

(b)

Figure 3: Transformation of the plane $(z)$ on the half plane $(\zeta)$.

and the impermeability condition of the barrier leads to

$$
X^{+}(\alpha, \beta)=\operatorname{Re} f^{+}\left(\frac{\alpha}{\varepsilon}-i \beta\right)=0, \quad \text { on the segment }(\alpha=0 ;-h \leq \beta \leq-l) .
$$

In the same way, we build in $D^{-}$a second analyticalcomplex variable function $z=\alpha / \varepsilon-i \beta$ as follows:

$$
f^{-}\left(\frac{\alpha}{\varepsilon}-i \beta\right)=1+\sum_{m=1}^{\infty} a_{1, m} \exp \left(m \frac{\pi}{h}\left(\frac{\alpha}{\varepsilon}-i \beta\right)\right)
$$

The continuity conditions at the border of the two domains and by the analytical extension allow the following equality:

$$
f^{+}\left(\frac{\alpha}{\varepsilon}-i \beta\right)=f^{-}\left(\frac{\alpha}{\varepsilon}-i \beta\right)=f\left(\frac{\alpha}{\varepsilon}-i \beta\right)
$$

The determination of local disturbances $a_{1, m}$ leads to find a function $f$, analytical in the domain $\{-\infty<\alpha<+\infty ;-h \leq \beta \leq 0\}$ without the segment $\{\alpha=0 ;-h \leq \beta \leq-l\}$. This function is bounded in this domain except at the singularity point. Furthermore, it verifies the following restrictions:

$$
\begin{gathered}
\operatorname{Im} f\left(\frac{\alpha}{\varepsilon}-i \beta\right)=0, \quad \text { on the boundaries } \beta=0, \beta=-h, \\
\operatorname{Re} f\left(\frac{\alpha}{\varepsilon}-i \beta\right)=0, \quad \text { on }[\alpha=0 ;-h \leq \beta \leq-l], \\
f\left(\frac{\alpha}{\varepsilon}-i \beta\right) \text { equal to unity at infinity. }
\end{gathered}
$$

According to [6], we can obtain a conform transformation of this domain on the superior halfplane (see Figure 3) and seek an analytic function satisfying the conditions on the boundaries in the later. We find, in particular, the value of $\delta=(\cos (\pi l / 2 h))^{-1}$ (see [6]).

By using the formula of Schwarz-Christofel [6], the conform transformation is written as

$$
z=-\frac{h}{\pi} \ln \left(\frac{\sqrt{\zeta^{2}-1}-\tan (\pi l / 2 h)}{\sqrt{\zeta^{2}-1}+\tan (\pi l / 2 h)}\right),
$$


and its inverse transformation is

$$
\zeta=\sqrt{1+\tan ^{2} \frac{\pi l}{2 h} \operatorname{coth}^{2} \frac{\pi z}{2 h}}
$$

The function $H(\zeta)=f(z)$ is holomorphic in the superior half-plane and also bounded except at the point $A_{3}^{\prime}$. On the real axis, this function verifies the following conditions:

$$
\begin{aligned}
& \operatorname{Im}[H(\zeta)]=0, \quad \text { on the segment }\left[\mathrm{A}_{6}^{\prime}, \mathrm{A}_{1}^{\prime}\right] ;\left[\mathrm{A}_{1}^{\prime}, \mathrm{A}_{2}^{\prime}\right], \\
& \operatorname{Re}[H(\zeta)]=0, \quad \text { on the segment }\left[\mathrm{A}_{2}^{\prime}, \mathrm{A}_{3}^{\prime}\right] ;\left[\mathrm{A}_{3}^{\prime}, \mathrm{A}_{4}^{\prime}\right], \\
& \operatorname{Im}[H(\zeta)]=0, \quad \text { on the segment }\left[\mathrm{A}_{4}^{\prime}, \mathrm{A}_{5}^{\prime}\right] ;\left[\mathrm{A}_{5}^{\prime}, \mathrm{A}_{6}^{\prime}\right] .
\end{aligned}
$$

By applying the formula of Signorini [6], we find the function

$$
H(\zeta)=\frac{1}{\sin (\pi l / 2 h)}\left(\frac{\sqrt{\zeta^{2}-1}}{\zeta}\right)
$$

and returning to the $z$-plane using (3.17), we finally find

$$
f\left(\frac{\alpha}{\varepsilon}-i \beta\right)=\left\{\sin \frac{\pi l}{2 h} \cdot \sqrt{1+\left[\frac{\tanh (\pi / 2 h)(\alpha / \varepsilon-i \beta)}{\tan (\pi l / 2 h)}\right]^{2}}\right\}^{-1} .
$$

This function describes the full flow in particular the field of the disturbances in the vicinity of the barrier. The coefficients $a_{1, m}$ can be calculated easily. In fact on the vertical $\alpha=0$, the function (3.20) is

$$
f(i \beta)=\sqrt{2} \frac{\cos (\pi \beta / 2 h)}{\sqrt{\cos (\pi \beta / h)-\cos (\pi l / h)}} .
$$

Furthermore the relations (3.10) to (3.14) permit the function

$$
f(i \beta)=1+\sum_{m=1}^{\infty} a_{1, m}\left(\cos m \frac{\pi \beta}{h}+i \sin m \frac{\pi \beta}{h}\right)
$$

where the coefficients $a_{1, m}$ are obtained by using the Fourier series expansion on the interval $[-h, 0]$.

It follows that

$$
a_{1, m}=\frac{2}{h} \int_{0}^{-h} f(i \beta) \cos m \frac{\pi \beta}{h} d \beta
$$


Thus

$$
a_{1, m}=P_{m}\left(\cos \frac{\pi l}{h}\right)+P_{m-1}\left(\cos \frac{\pi l}{h}\right)
$$

where $P_{m}$ and $P_{m-1}$ are the Legendre polynomials of degree $m$ and $m-1$, respectively, in the third integral representation.

The $P_{m}$ formula is given below:

$$
\begin{aligned}
P_{m}\left(\cos \frac{\pi l}{h}\right)=2 \frac{1.3 .5 \cdots(2 m-1)}{2.4 .6 \cdots 2 m}\{ & \cos m \frac{\pi l}{h}+\frac{1 \cdot m}{1 \cdot(2 m-1)} \cos (m-2) \frac{\pi l}{h} \\
& \left.+\frac{1.3 \cdot m(m-1)}{1.2 \cdot(2 m-1)(2 m-3)} \cos (m-4) \frac{\pi l}{h}+\cdots\right\} .
\end{aligned}
$$

Using (3.1), (3.2), (3.4), (3.8), and (3.14), the horizontal and vertical components of the displacement of the flow, in all domain $D$, can be written as follows:

$$
\begin{gathered}
X_{1}(\alpha, \beta, \tau)=X_{e}(\alpha-\tau)+X_{e}(-\tau) \sum_{m=1}^{\infty} a_{1, m} \cos m \frac{\pi \beta}{h} \exp \left(-\frac{m \pi|\alpha|}{\varepsilon h}\right), \\
Y_{1}(\alpha, \beta, \tau)=X_{e}(-\tau) \sum_{m=1}^{\infty} a_{1, m} \sin m \frac{\pi \beta}{h} \exp \left(-\frac{m \pi|\alpha|}{\varepsilon h}\right),
\end{gathered}
$$

where $X_{1}$ and $Y_{1}$ are, respectively, the horizontal and vertical components of the displacement of the particle.

Remark 3.1. According to (3.1), (3.2), and (3.26), one notices that displacements $X_{1}(\alpha, \beta, \tau)$ and $Y_{1}(\alpha, \beta, \tau)$ are the independent contributions of the parts "wave" and "local disturbances" of the flow. On the first order of approximation, the part "wave" of the flow imposes a uniform distribution of displacements and speeds on a vertical. The part "disturbances local" permits to take into account the presence of the obstacle.

\section{Application}

For illustration, we consider as an example a solitary wave (see [5]) $X_{e}(\alpha-\tau)$ which is emitted at the upstream at time $\tau=0$ and arrives exactly above the barrier at time $\tau=\tau_{0}$ :

$$
X_{e}(\alpha-\tau)=\operatorname{\varepsilon h}\left\{1-\tanh \left[\frac{3}{4 h}\left(\alpha-\left(\tau-\tau_{0}\right)\right)\right]\right\}
$$

Now, we calculate the velocity field and the local disturbances of the flow. We observe that the horizontal displacement is independent of the variable $\beta$ in accordance with the theory of long gravity waves. 
The derivative of the expression (3.26) with respect to $t$ gives the horizontal and vertical components, respectively, $u_{1}$ and $v_{1}$ of the velocity field

$$
\begin{aligned}
u_{1}= & \frac{3}{4} \varepsilon^{2} \sqrt{g h}\left\{1-\tanh ^{2}\left[\frac{3}{4 h}\left(\alpha-\left(\tau-\tau_{0}\right)\right)\right]\right\} \\
& +\frac{3}{4} \varepsilon^{2} \sqrt{g h} \times\left\{1-\tanh ^{2}\left[\frac{3}{4 h}\left(\tau-\tau_{0}\right)\right]\right\} \sum_{m=1}^{\infty} a_{1, m} \cos m \frac{\pi \beta}{h} \exp \left(-\frac{m \pi|\alpha|}{\varepsilon h}\right) \\
v_{1}= & \frac{3}{4} \varepsilon^{2} \sqrt{g h}\left\{1-\tanh ^{2}\left[\frac{3}{4 h}\left(\tau-\tau_{0}\right)\right]\right\} \sum_{m=1}^{\infty} a_{1, m} \sin m \frac{\pi \beta}{h} \exp \left(-\frac{m \pi|\alpha|}{\varepsilon h}\right) .
\end{aligned}
$$

We observe that the horizontal (vertical) components of the velocity is symmetric, that is, $u_{1}(\alpha, \beta)=u_{1}(-\alpha, \beta)$ (resp., antisymmetric, i.e., $v_{1}(\alpha, \beta)=-v_{1}(-\alpha, \beta)$ ).

From (4.2), we deduce the horizontal and vertical components, respectively, $u_{1}^{*}$ and $v_{1}^{*}$ of the field local disturbances

$$
\begin{aligned}
& u_{1}^{*}=\frac{3}{4} \varepsilon^{2} \sqrt{g h}\left\{1-\tanh ^{2}\left[\frac{3}{4 h}\left(\tau-\tau_{0}\right)\right]\right\} \sum_{m=1}^{\infty} a_{1, m} \cos m \frac{\pi \beta}{h} \exp \left(-\frac{m \pi|\alpha|}{\varepsilon h}\right), \\
& v_{1}^{*}=\frac{3}{4} \varepsilon^{2} \sqrt{g h}\left\{1-\tanh ^{2}\left[\frac{3}{4 h}\left(\tau-\tau_{0}\right)\right]\right\} \sum_{m=1}^{\infty} a_{1, m} \sin m \frac{\pi \beta}{h} \exp \left(-\frac{m \pi|\alpha|}{\varepsilon h}\right) .
\end{aligned}
$$

The components $u_{1}^{*}$ and $v_{1}^{*}$ can be written as

$$
\begin{aligned}
& u_{1}^{*}=c(\tau) \cdot u_{1}^{* *}(\alpha, \beta), \\
& v_{1}^{*}=c(\tau) \cdot v_{1}^{* *}(\alpha, \beta),
\end{aligned}
$$

where

$$
\begin{gathered}
c(\tau)=\frac{3}{4} \varepsilon^{2} \sqrt{g h}\left\{1-\tanh ^{2}\left[\frac{3}{4 h}\left(\tau-\tau_{0}\right)\right]\right\}, \\
u_{1}^{* *}(\alpha, \beta)=\sum_{m=1}^{\infty} a_{1, m} \cos m \frac{\pi \beta}{h} \exp \left(-\frac{m \pi|\alpha|}{\varepsilon h}\right), \\
v_{1}^{* *}(\alpha, \beta)=\sum_{m=1}^{\infty} a_{1, m} \sin m \frac{\pi \beta}{h} \exp \left(-\frac{m \pi|\alpha|}{\varepsilon h}\right) .
\end{gathered}
$$

Equations (4.4) show that the coefficient $c(\tau)$ intervenes only as a parameter of scale of the local disturbances (i.e., the kinematic of the flow remains unchanged qualitatively whatever the position of the wave relatively to the barrier). Naturally the intensity of the local disturbances is maximum at the moment $t=t_{0}$. 


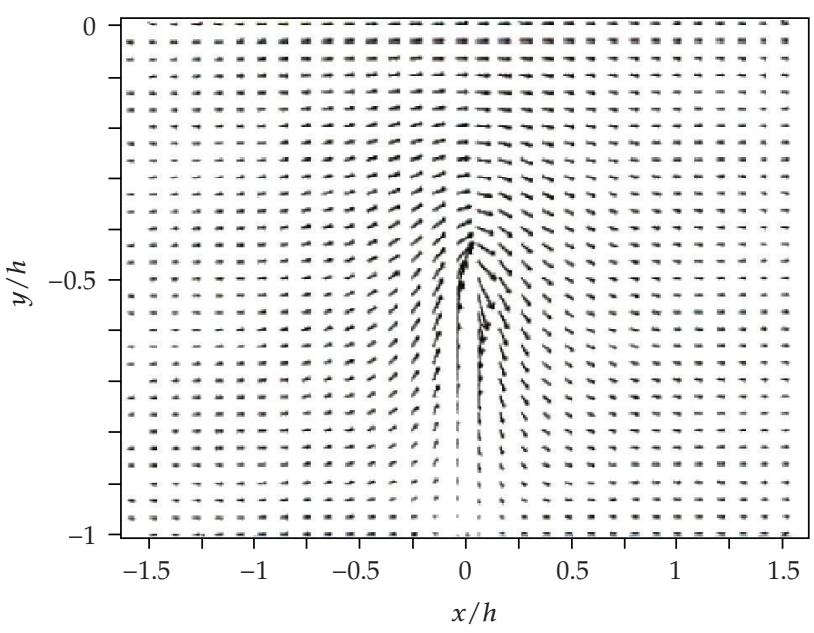

Figure 4: The field velocity $h=20 \mathrm{~cm}, l=10 \mathrm{~cm}$, and $\varepsilon=0.6$.

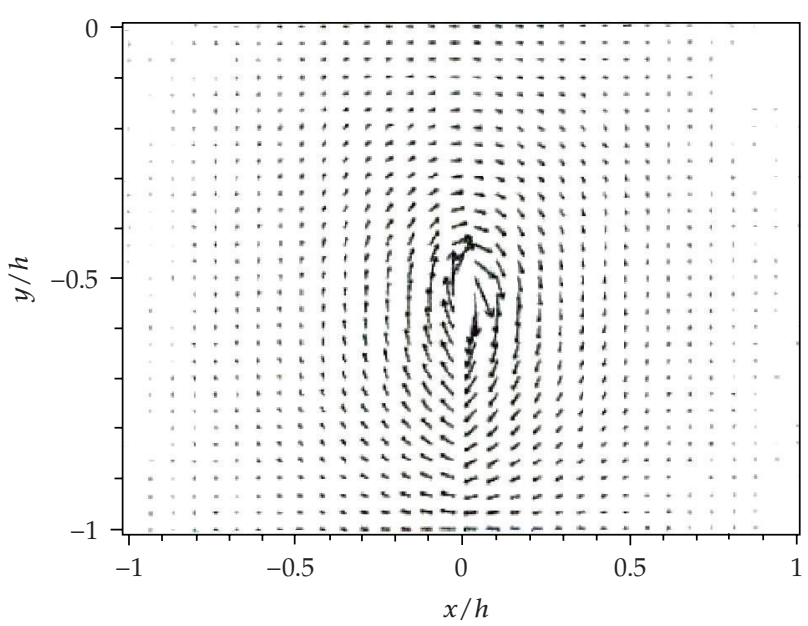

Figure 5: The local disturbances $h=20 \mathrm{~cm}, l=10 \mathrm{~cm}$, and $\varepsilon=0.6$.

\subsection{Numerical simulation}

The numerical simulation is realized on the Apollo station by using the subprogram of the Legendre polynomials. The data used are $h=20 \mathrm{~cm}, l=10 \mathrm{~cm}$, and $\varepsilon=0.6$. The maximum velocity of incident flow is approximately $38 \mathrm{~cm} / \mathrm{s}$. We note that the series (3.26) converge slowly, for this, we have used $510^{3}$ terms. The criterionof convergence is the impermeability condition of thebarrier (i.e., the norm of the horizontal component remains within a specified tolerance $10^{-4}$ ).

The graphical representation of the field of velocity and the local disturbances are given, respectively.

The shallow water theory at the first order of approximation permits to determine in a simple way the velocity field and the local disturbances when the interaction of a solitary wave occurs with an isolated obstacle. The numerical simulation illustrates the flow 
at the vicinity of the barrier and shows, in particular, the zone of the influence of the local disturbances.

In a future work, we intend to study the problem by using the shallow water theory at the second order of approximation in order to consider, further, the effects of the reflected wave on the kinematic flow barrier.

\section{Acknowledgment}

The work simulation is realized in the Computer Center of the Laboratoire des Ecoulements Géophysiques et Industriels (L.E.G.I), Joseph Fourier University of Grenoble (France).

\section{References}

[1] E. Barthélemy, A. Kabbaj, and J.-P. Germain, "Long surface wave scattered by a step in a two-layer fluid," Fluid Dynamics Research, vol. 26, no. 4, pp. 235-255, 2000.

[2] W. R. Dean, "On the reflexion of surface waves by a submerged plane barrier," Proceedings of the Cambridge Philosophical Society, vol. 41, pp. 231-238, 1945.

[3] J.-P. Germain, "Théorie générale des mouvements d'un fluide parfait pesant en eau peu profonde de profondeur constante," Comptes Rendus Hebdomadaires des Séances de l'Académie des Sciences. Séries A et B, vol. 274, pp. A997-A1000, 1972.

[4] L. Gulli, Etude du passage d'une houle en eau-peu-profonde sur une barrière verticale immergée, M.S. thesis, Universite de Grenoble, Grenoble, France, 1975.

[5] F. J. Seabra-Santos, D. P. Renouard, and A. M. Temperville, "Numerical and experimental study of the transformation of a solitary wave over a shelf or isolated obstacle," The Journal of Fluid Mechanics, vol. 176, pp. 117-134, 1987.

[6] A. Guerziz and A. Laouar, "Passage of a long wave over a vertical barrier: calculation of the local disturbances by a complex variable method," International Journal of Mathematics and Computer Science, vol. 1, no. 3, pp. 361-367, 2006. 


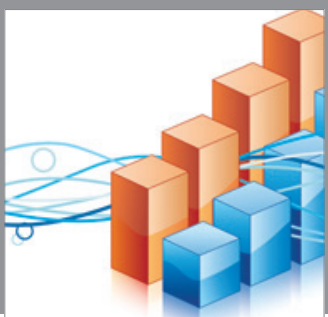

Advances in

Operations Research

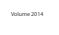

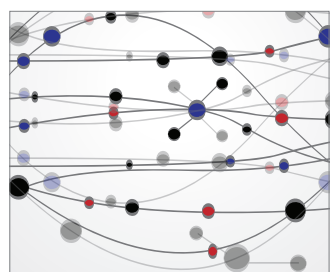

\section{The Scientific} World Journal
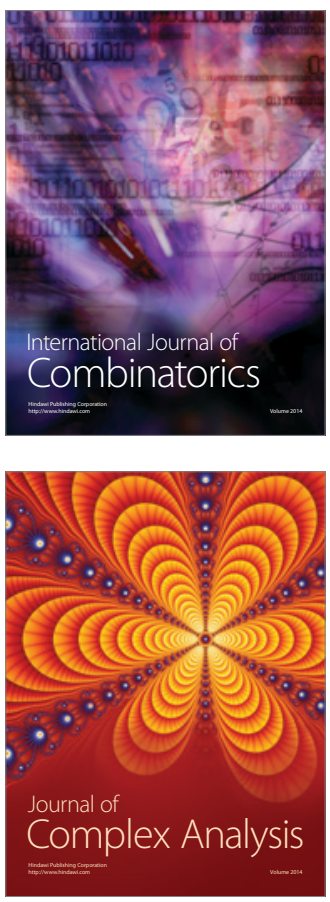

International Journal of

Mathematics and

Mathematical

Sciences
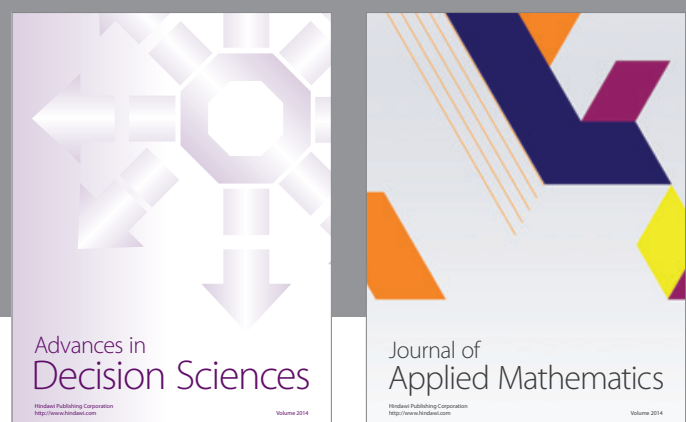

Journal of

Applied Mathematics
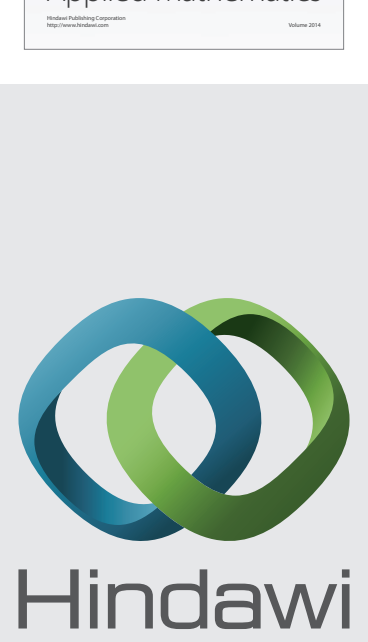

Submit your manuscripts at http://www.hindawi.com
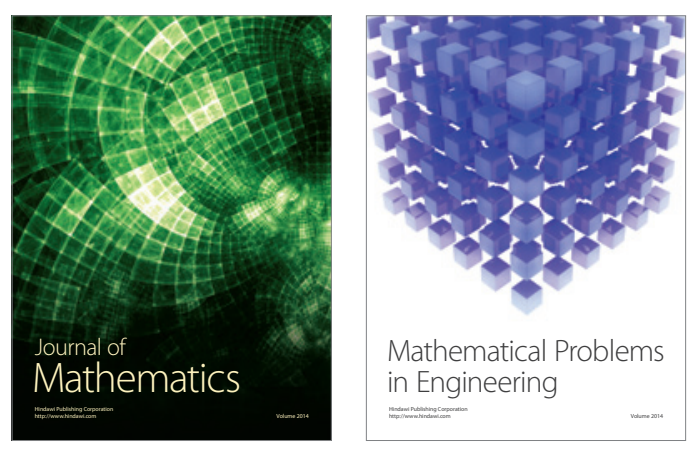

Mathematical Problems in Engineering
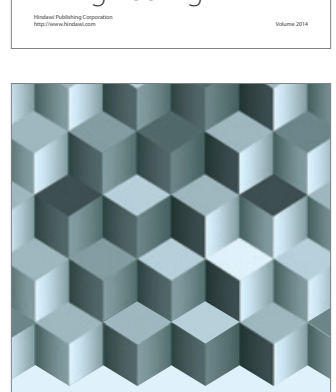

Journal of

Function Spaces
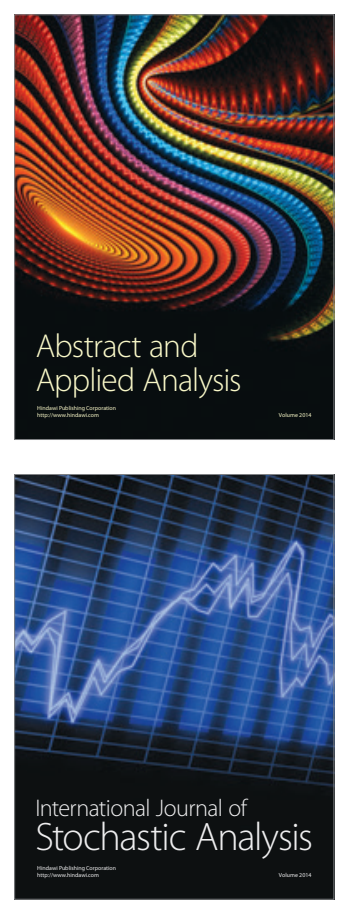

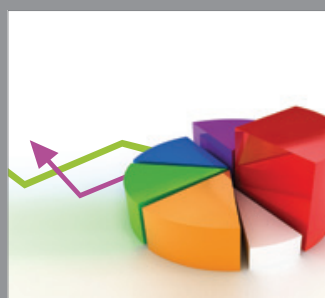

ournal of

Probability and Statistics

Promensencen
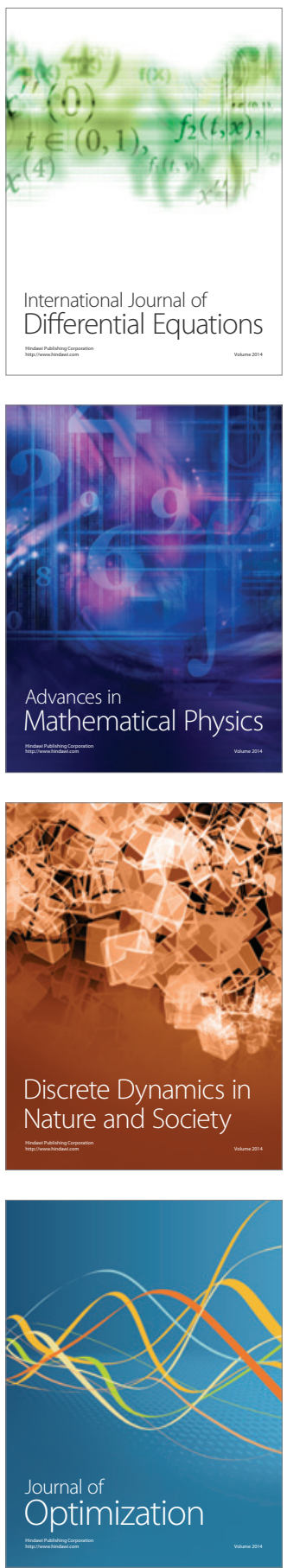